\title{
Bacterial community characterization of the rhizobiome of plants belonging to Solanaceae family cultivated in desert soils
}

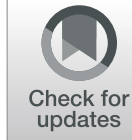

\author{
Aarón Barraza ${ }^{1}$, María Goretty Caamal-Chan ${ }^{1}$, Thelma Castellanos ${ }^{2}$ and Abraham Loera-Muro ${ }^{1 *}$ (D)
}

\begin{abstract}
Purpose: The plant Solanaceae family is one of the most important for global agriculture and nutrition. Within this plant family, two plant species stand out for their economic importance and for human consumption, which are tomato (Solanum lycopersicum) and chili pepper (Capsicum annuum). Moreover, those plants support diverse and characteristic microbial communities that are uniquely suited to the plant habitat and intimately connected to plant health. The main objective of this work is the bacterial community characterization in the rhizobiome of tomato and chili pepper, cultivated in arid environments.

Methods: Five crop fields located in the south of the peninsula of Baja California, Mexico, were sampled. Total DNA was extracted from rhizosphere, rhizoplane, and endophytic root compartment and sequenced by lllumina MiniSeq platform technology applied to $16 \mathrm{~S}$ rRNA gene V3 region.

Results: We were able to obtain 1,195,426 total reads and 1,725,258 total reads for tomato and chili pepper samples, respectively. The analysis of the bacterial community structures confirmed that the two plant species showed differences in their microbial community structures. Nonetheless, the microbial community structures were directly and equally influenced by the crop field localization of each plant species. Interestingly, we determined that in both plant species, the Proteobacteria was the main phylum.

Conclusion: In conclusion, we found that several bacterial families are part of the core rhizobiome (28 OTUs) for both tomato and chili pepper, but the most abundant were the Pseudomonadaceae family and the Pseudomonas genus, which most probably play a pivotal role in the microbial ecology to benefit both crop plants.
\end{abstract}

Keywords: Capsicum annuum, Solanum lycopersicum, Rhizobiome, Rhizosphere, Rhizoplane, Endophytic root bacteria

\section{Introduction}

Plants are capable to support diverse microbial communities. Those are specific to the plant habitat and are intimately coupled with plant requirements. Those microbial communities are species, and sometimes, genotype-specific (Allard et al. 2016). The plant bacterial communities contribute to crop productivity by plant

\footnotetext{
* Correspondence: aloera@cibnor.mx

'CONACYT-Centro de Investigaciones Biológicas del Noroeste, SC, Instituto Politécnico Nacional 195, Playa Palo de Santa Rita Sur, C.P. 23096, La Paz, B.C.S., Mexico

Full list of author information is available at the end of the article
}

growth promotion and biocontrol of phytopathogens, therefore into ecofriendly agriculture (Posada et al. 2018). Microbiota encompasses several functional contexts: stimulation of seed germination, plant growth, and resistance promotion to abiotic and biotic stresses (Kalam et al. 2017; Larousse et al. 2017). Previous reports have been shown that the microbial communities associated with diverse plant hosts, tissue, geographic location, and season are all able to influence directly onto bacterial community structure composition. Albeit, the specific extent to which each factor has played an effect has varied from study to study (Coleman-Derr et al.

(c) The Author(s). 2020 Open Access This article is licensed under a Creative Commons Attribution 4.0 International License, which permits use, sharing, adaptation, distribution and reproduction in any medium or format, as long as you give appropriate credit to the original author(s) and the source, provide a link to the Creative Commons licence, and indicate if changes were made. The images or other third party material in this article are included in the article's Creative Commons licence, unless indicated otherwise in a credit line to the material. If material is not included in the article's Creative Commons licence and your intended use is not permitted by statutory regulation or exceeds the permitted use, you will need to obtain permission directly from the copyright holder. To view a copy of this licence, visit http://creativecommons.org/licenses/by/4.0/. 
2016). The microbial community associated with roots known as "rhizobiome" was proposed to be assembled in two steps: (i) the rhizosphere is colonized by a subset of the bulk soil community and (ii) the rhizoplane and the endosphere are colonized by a subset of the rhizosphere community. All those plant-associated microorganisms together establish a core microbiome in the rhizobiome. The core microbiome consisting of bacterial groups is common to the species (Olanrewaju et al. 2018; Sasse et al. 2018; Ávila et al. 2019). The rhizosphere (defined as microbes living in the zone around root (Sasse et al. 2018)) is rich in nutrients due to the accumulation of plant exudates, and these contain amino acids and sugars that provide a nitrogen and carbon source required for colonizing bacteria. This induces the bacterial community to colonize the rhizoplane and rhizosphere (Qiao et al. 2017; Posada et al. 2018). The rhizoplane microorganisms (defined as root surface adhered microbes (Sasse et al. 2018)) can metabolize essential inorganic nutrients (P, S, Mg, Mn, and $\mathrm{Cu}$ ) directly from minerals, fixing atmospheric nitrogen, and then promoting plant growth (Lopez et al. 2011). Finally, some bacteria from the rhizoplane can colonize intercellular spaces in several internal plant tissues without causing any harm or disease, and some bacteria enhance plant growth and increase plant resistance to pathogens; those bacteria are collectively known as endophytic bacteria (defined as microbe living within plant tissue in the endosphere (Sasse et al. 2018)) (Lopez et al. 2011; Passari et al. 2016).

Solanaceae is one of the most important plant families for global agriculture. Among the different solanaceous species, tomato (Solanum lycopersicum) and chili pepper (Capsicum annuum) are two major crops of outstanding importance worldwide (Haak et al. 2014; Seguí-Simarro 2016). Chili peppers can be used as a spice, vegetable, and food coloring, in addition to pharmaceutical applications. In Mexico, which is the center of origin, its production was estimated in more than two million tons with a market value of more than one billion dollars (SIAP 2016; Barchenger et al. 2018). Otherwise, tomato is also an important crop since its production is more than three million tons with a market value of approximately one billion dollars (SIAP 2016). The importance of tomato lies in its content of vitamin C, potassium, folic acid, lycopene and other carotenoids, and several phytochemicals with pharmaceutical potential (Perveen et al. 2015).

Up to today, the global human population is projected to reach up to 9 billion people by 2050, with an increase in food demand by 1.7-fold. Furthermore, the increase in food production must be achieved by a reduction of supply cost for agriculture, since up to $50 \%$ of crop production arises from N-based fertilizers (Camilios-Neto et al. 2014). Otherwise, arid and semi-arid regions represent one third of the Earth's land surface area. These ecosystems are characterized by their low water availability which restrains biological activity. Plants in these habitats are subjected to many types of abiotic stress including extreme temperature fluctuations, high ultraviolet radiation, low nutrient content soils, and drought (Fonseca-García et al. 2016). Arid, semi-arid, and Mediterranean-type regions currently support agricultural production for close to a third of the world's population (Mickan et al. 2019). Soil microorganisms in semi-arid regions are commonly subject to seasonal water stress; some groups of organisms are better able to adapt to soil water deficits than others by activating strategies such as dormancy, among others (Manzoni et al. 2012). Thus, the whole shared microbiome ("core") and those microorganisms that are shared by some individuals in the same growth conditions ("specific") of plants microbiome members are compared. That is the species/genotypes of microorganisms that are grown in the same environment in several plants or the species/ genotypes of microorganisms grown in many environments for a specific plant (Lebeis 2014; Tian et al. 2015). It is of utmost importance to improve agricultural production processes sustainably by developing better biofertilizers that help crops adapt to the increasingly extreme conditions that occur in different parts of the world.

Metagenomic analysis and comparison of the plantassociated microbiome have successfully led to a novel phylogenetic and functional insight in the plant microbiome and their interactions with host plants (Tian et al. 2015). The objective of this work is to study the core microbiome of the rhizobiome (rhizosphere, rhizoplane, and endophytic root compartment) of plant solanaceous species of chili pepper (C. annuum) and tomato (S. lycopersicum), grown in arid environments for a later comparative analysis between both rhizobiome. The above would allow finding which microorganism group is conforming to the Solanaceae family core rhizobiome, which group is specific for chili or tomato, and which are location specific. Altogether, it will allow a deeper understanding of the plant-microbe interaction to achieve an adaptation to their environment and that benefiting agricultural practices to develop specific and better biofertilizers for arid and semi-arid regions.

\section{Materials and methods}

Plant material sampling

Tomato (S. lycopersicum var. cerasiforme) and chili pepper (C. annuum var. annuum) samples (roots, soil attached to roots, and soil) were collected from five crop fields located in the southern region of the Baja California peninsula where the arid environment predominates: (i) La Matanza: 23.65.52 N, - 110.26.72 W; 
(ii) Todos Santos: 23.46.68 N, - 110.21.59 W; (iii) Comitán: 24.06.10 N, - 110.21.05 W; (iv) Pescadero: 23.36.67 $\mathrm{N},-110.19 .04 \mathrm{~W}$; and (v) Los Planes: 23.95.57 N, 109.93.75 W. All samples were collected in the production period of 2017-2018. Roots were excised from the plant stem and placed in plastic bags with soil attached. Three crop fields for tomato (La Matanza, Pescadero, Los Planes) and three crop fields for chili pepper (Comitán, La Matanza, Todos Santos) were sampled. Three samples per crop field were collected for both tomato and chili pepper. Fifty-four samples were obtained (27 for tomato and 27 for chili pepper) and designated as rhizoplane (9 samples), rhizosphere (9 samples), and root endophytic compartment (9 samples) for each plant. Those samples were pooled in 3 samples of rhizoplane, 3 samples of rhizosphere, and 3 samples of root endophytic compartment, for each plant, totaling 18 samples for sequencing ( 9 for tomato and 9 for chili pepper) (Fonseca-García et al. 2016). All samples were processed immediately for total DNA extraction.

\section{Sample preparation and DNA extraction}

We collected soil around the root $(\sim 15 \mathrm{~cm}$, rhizosphere) in $50 \mathrm{~mL}$ sterile tubes, and the soil attached to root samples (rhizoplane) were washed with sterile distilled water several times and vortexed for $5 \mathrm{~min}$ to obtain all the bacteria (including bacterial biofilms) (Kalam et al. 2017). After, to obtain the endophytic root sample, root tissue samples were sterilized (with $70 \%$ ethanol solution for $2 \mathrm{~min}$, then washed with sterile and distilled water for $5 \mathrm{~min}$, then washed with $5 \%(\mathrm{v} / \mathrm{v})$ sodium hypochlorite for $10 \mathrm{~min}$, and finally twice with sterile and distilled water) (Desgarennes et al. 2014). From rhizosphere and rhizoplane solutions, the microbial pellet was obtained by centrifugation at $3000 \mathrm{rpm}$ for 5 min. Root sterilized samples were powdered with mortar and pestle using liquid nitrogen and stored at $-80{ }^{\circ} \mathrm{C}$ until DNA extraction.

Total DNA was extracted as follows: starting from $500 \mathrm{mg}$ of soil (rhizoplane and rhizosphere) samples and root tissue (root endophytic compartment) with $1 \mathrm{~mL}$ of pre-warm extraction buffer (2\% SDS, $1.4 \mathrm{M} \mathrm{NaCl}, 100$ $\mathrm{mM}$ Tris, and $20 \mathrm{mM}$ EDTA). The samples were incubated for $1 \mathrm{~h}$ at $55{ }^{\circ} \mathrm{C}$ and then allowed to incubate for up to $72 \mathrm{~h}$ at room temperature. Then, $1 \mathrm{~mL}$ of the supernatant was taken and transferred into a $1.5-\mathrm{mL}$ new tube, and 1 volume of phenol:chloroform:isoamyl alcohol (25:24:1) pH 8.0 (Sigma-Aldrich, Saint Louis, MO, USA) was added, mixed, and centrifuged at 12 , $000 \times g$ 's for $15 \mathrm{~min}$. The resulting aqueous phase was transferred into $1.5 \mathrm{~mL}$ new tubes, and total DNA was precipitated with $500 \mu \mathrm{L}$ of isopropanol for $24 \mathrm{~h}$ at -20 ${ }^{\circ} \mathrm{C}$. The samples were centrifuged at $12,000 \times g$ 's for 10 min, and the supernatant was discarded. The samples were washed with $70 \%(\mathrm{v} / \mathrm{v})$ ethanol. The total DNA pellet was air-dried and resuspended in $50 \mu \mathrm{l}$ of buffer TE (10:1). Total DNA was visualized on an agarose gel to ensure the integrity and measured in a NanoDrop 2000 system (Thermo Fisher Scientific, Waltham, MA, USA) to determine the quality (ratio of $\lambda 260 \mathrm{~nm} / 280 \mathrm{~nm}$ ) for all samples. Finally, total DNA samples were stored at $20{ }^{\circ} \mathrm{C}$ until sequencing.

\section{S rDNA V3 region PCR amplification and sequencing}

Total DNA was processed as follows: PCR amplification of $16 \mathrm{~S}$ rDNA V3-V4 region was carried out using V3$338 \mathrm{f}$ and V4-806r universal primers. The resulting PCR amplification product was used as a template to amplify only the V3 region using V3-338f and V3-533r primers with Illumina adapters, and indices were also added following the manufacturer's recommendations (Illumina, San Diego, CA, USA). The resulting V3 region-amplified products were quantified in a Qubit (Thermo Fisher Scientific, Waltham, MA, USA) to obtain an equimolar pool for each sample. Sequencing reads were generated using the $2 \times 150$ (300 cycles) base-read length chemistry of the Illumina MiniSeq platform.

\section{Bioinformatics and statistical analysis}

Sequencing reads generated were filtered for quality control $(Q \geq 33)$ using the modified Mott algorithm, paired-end, and merged with BBmerge to obtain sequence reads of tomato and chili pepper samples. Those sequence reads were then assembled with a minimum identity of $98 \%$, and both contigs and unassembled reads were compared against the GenBank non-redundant database using Megablast. Megablast results were used to create a curated database specific for each sample. Sequence reads per sample were clustered against its corresponding created database using Sequence Classifier to obtain operational taxonomic unit (OTU) frequencies or OTU tables using Geneious Prime (www.geneious.com). These OTU tables were then processed by " $R$ " programming language using a variety of packages and custom scripts (www.r-project.org). We estimated Chao1, Shannon, and Simpson $\alpha$-biodiversity indices. Venn's diagrams were plotted using the package "VennDiagram." Bray-Curtis distance estimations were calculated using the "vegdist" function with the package "vegan," as well as principal component analysis using "prcomp" function with the package "ggfortify" (Oksanen et al. 2012; Coleman-Derr et al. 2016; Castañeda and Barbosa 2017). PERMANOVA statistical analysis was performed with "adonis" function with the package "vegan." To identify the OTUs that were enriched or depleted as a function of the microbial environment (soil, rhizosphere, and root endophytic compartment), differentially abundant OTUs (DAOTUs) were evaluated by fitting a generalized linear 
model (GLM) with a negative binomial distribution (Poudel et al. 2019). Likelihood ratio tests and contrast analyses were performed on the fitted GLM to identify DAOTUs. The OTU counts from soil were used as a control and compared with root endophytic compartment and rhizosphere samples in a contrast analysis. All tests were adjusted to soil (control) for the falsediscovery rate $(\mathrm{FDR})(P<0.05)$ using the BenjaminiHochberg method. General community profiles were constructed using OTUs labeled at the phylum level and visualized in a bar plot graph.

Finally, sequencing data were deposited in the National Center for Biotechnology Information (NCBI) Sequence Read Archive (SRA) database under BioProject accession number PRJNA562381.

\section{Results}

\section{Sequencing run metrics}

The total DNA samples (root endophytic compartment, rhizosphere, and rhizoplane) of C. annuum and S. lycopersicum were sequenced by the $16 \mathrm{~S}$ rDNA V3 region with the Illumina MiniSeq $(2 \times 150,300$ cycles $)$ platform. The NGS sequencing yielded $~ 2.9$ million raw reads for the 18 samples analyzed. For tomato samples, $1,195,426$ raw reads (on average 123,333 sequences per sample) were acquired (Table 1). These data were further processed for control quality analysis (trimming, filtering, pairing-end, and merging) to obtain $554,999 \mathrm{se}-$ quences and classified into 6529 OTUs, for which $82.41 \%$ were classified and $17.59 \%$ were not classified into any known OTU reported in the "nt-nr" database (GenBank). In chili pepper, 1,725,258 raw reads (on average 166,003 sequences per sample) were acquired (Table 1). After sequence processing, we obtained 758, 362 sequences classified into 6244 OTUs, from which $89.89 \%$ of the processed sequences were assigned into a known OTU and $10.11 \%$ were not classified into any known OTU reported in the " $n t-n r$ " database (GenBank). For both totaling 12,773 OTUs, the average paired-end sequence length was $\sim 185 \mathrm{bp}$ after merging.

\section{Diversity of communities associated with C. annuum and \\ S. lycopersicum}

The microbial community diversity for rhizosphere, rhizoplane, and root endophytic compartment samples from three crop fields sampled for each crop plant Solanaceae species (C. annuum and S. lycopersicum) was assessed through alpha indices estimations. Interestingly, we were able to determine that for both Solanaceae species, the alpha-diversity (observed OTUs, Chao1 index, and Shannon index) indices were greater for both the root endophytic compartment and the rhizoplane samples than those determined for the rhizosphere samples (Fig. 1, Tables 1 and 2). Moreover, when these alpha-
Table 1 Sequencing reads generated, filtered for the quality control $(Q \geq 33)$, and assembled with a minimum identity of 98\%, for comparison against the GenBank non-redundant database using Megablast

\begin{tabular}{lllll}
\hline Samples & Reads & QC 33 & Classified (\%) & Unclassified (\%) \\
\hline C. annuum & & & & \\
CCR & 476624 & 416578 & 88.67 & 11.33 \\
CCSR & 94578 & 81808 & 91.76 & 8.24 \\
CCS & 1246008 & 129392 & 74.98 & 25.02 \\
CMR & 171288 & 150112 & 96.81 & 3.19 \\
CMSR & 91774 & 80626 & 96.13 & 3.87 \\
CMS & 116478 & 103222 & 98.73 & 1.27 \\
CTR & 223200 & 196626 & 79.6 & 20.4 \\
CTSR & 192752 & 169662 & 98.64 & 1.36 \\
CTS & 212736 & 188698 & 90.63 & 9.37 \\
S. IyCopersicum & & & & \\
TMR & 192064 & 167744 & 82.48 & 17.52 \\
TMSR & 76236 & 69698 & 87.57 & 2.43 \\
TMS & 159230 & 150390 & 92.9 & 7.1 \\
TPR & 131974 & 123956 & 48 & 52 \\
TPSR & 67318 & 64358 & 99.45 & 0.55 \\
TPS & 174164 & 163012 & 92.16 & 7.84 \\
TLR & 84630 & 78984 & 40.94 & 59.06 \\
TLSR & 82800 & 78432 & 99.25 & 0.75 \\
TLS & 227010 & 213424 & 86.63 & 13.37 \\
\hline CCR & & & \\
\hline
\end{tabular}

CCR chili pepper, Comitán endophytic root; CCSR chili pepper, Comitán, rhizosphere; CCS chili pepper, Comitán, rhizoplane; CMR chili pepper, La Matanza, endophytic root; CMSR chili pepper, La Matanza, rhizosphere; CMS chili pepper, La Matanza, rhizoplane; CTR chili pepper, Todos Santos, endophytic root; CTSR chili pepper, Todos Santos, rhizosphere; CTS chili pepper, Todos Santos, rhizoplane; TMR tomato, La Matanza, endophytic root; TMSR tomato, La Matanza, rhizosphere; TMS tomato, La Matanza, rhizoplane; TPR tomato, Pescaderos, endophytic root; TPSR tomato, Pescaderos, rhizosphere; TPS tomato, Pescaderos, rhizoplane; TLR tomato, Los Planes, endophytic root; TLSR tomato, Los Planes, rhizosphere; TLS tomato, Los Planes, rhizoplane

diversity indices were examined by crop field localization, we also determine that alpha-diversity followed a peculiar trend of greater indices for crop fields located near the sea shores (Comitán and La Matanza) than those crop fields afar the coast (Pescadero, Todos Santos, Los Planes) (Fig. S1, Table S1).

The bacterial diversity, richness, and evenness analyses (observed OTUs and Chaol index, Shannon index, and Simpson index, respectively) were carried out to establish bacterial community structures based on several approaches such as plant Solanaceae species (C. annuum and $S$. lycopersicum), plant species and type of samples (rhizoplane, rhizosphere, and root endophytic compartment), type of samples, and crop field locations. In the first approach, no significant differences were found between the species for any diversity, richness, and evenness indices (Fig. S3). We analyzed the differences 
a)
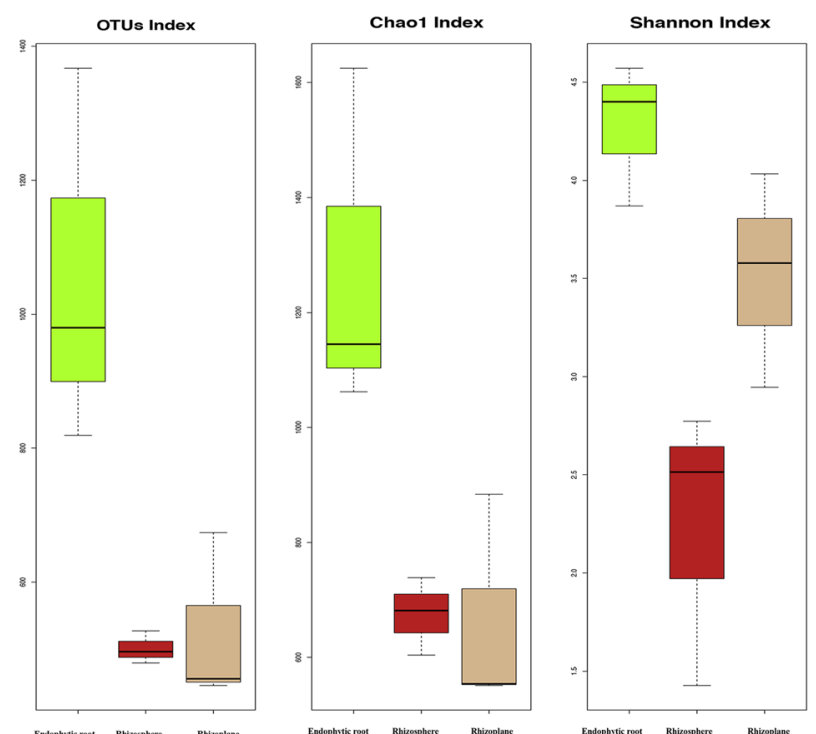

b)
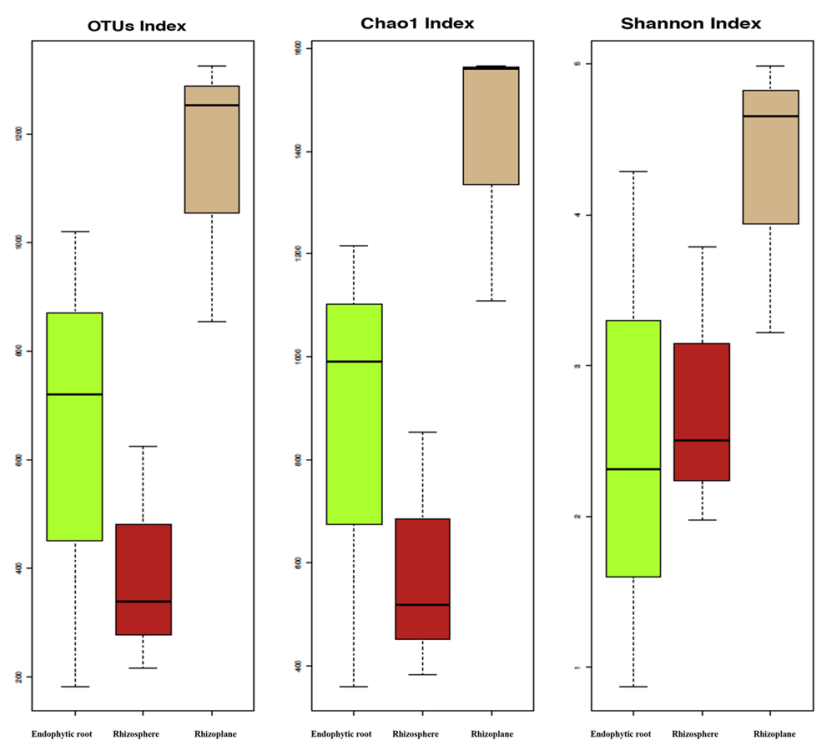

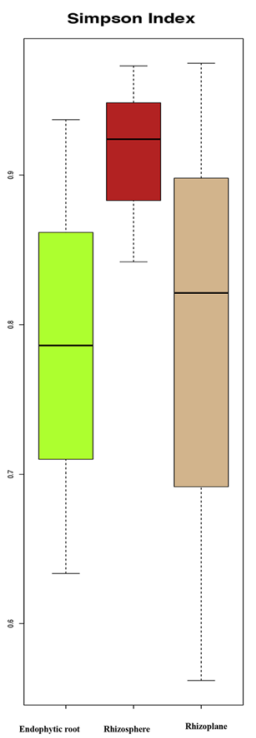

Fig. 1 Alpha-diversity index of bacterial communities across the different samples of a C. annuum and $\mathbf{b}$ S. lycopersicum

Table 2 Alpha-diversity indices of bacterial communities across the different samples

\begin{tabular}{|c|c|c|c|c|}
\hline Biodiversity indices & OTUs & Chao-1 & Shannon & Simpsor \\
\hline \multicolumn{5}{|l|}{ C. annuum } \\
\hline Root endophytic compartment & 1055 & 1277.34 & 4.28 & 0.786 \\
\hline Rhizosphere & 500 & 674.67 & 2.24 & 0.913 \\
\hline Rhizoplane & 525 & 663.46 & 3.52 & 0.786 \\
\hline \multicolumn{5}{|l|}{ S. lycopersicum } \\
\hline Root endophytic compartment & 640 & 1277.34 & 2.49 & 0.359 \\
\hline Rhizosphere & 391 & 674.67 & 2.76 & 0.975 \\
\hline Rhizoplane & 1144 & 663.46 & 4.29 & 0.821 \\
\hline
\end{tabular}


between both plants and by sample type (rhizoplane, rhizosphere, and root endophytic compartment); we found significant differences in the diversity indices (observed OTUs and Chao1 index) only for rhizoplane samples between $C$. annuum and S. lycopersicum $(P<0.05)$ (Fig. S4a, b). In the analysis by sample type and both plant species treated as a single plant family, we were able to determine that root endophytic compartment samples showed the highest estimations of diversity (observed OTUs and Chao1 index) and richness (Shannon index). The rhizosphere samples showed the lowest diversity and richness estimations, except for the evenness estimation which was the highest (Fig. S5). Finally, when we analyzed the five crop field locations for diversity, richness, and evenness estimations, we did not determine any differences, in spite of the geographical location differences and the agronomical practices applied to each crop field (Fig. S6).

We proceeded to determine the differences between root endophytic compartment samples, rhizosphere samples, and rhizoplane samples from $C$. апnиum crop fields through beta-diversity analysis. We were able to determine that the microbial community structures for the root endophytic compartment samples and in the rhizoplane were quite similar, regardless of the location where each sample came from by a hierarchical clustering analysis based on a Bray-Curtis distance estimation matrix (Fig. 2a). Also, when we carry out an unsupervised hierarchical biclustering analysis of the most abundant OTUs (> 100 reads/sample) for C. annuum samples, we found that samples from La Matanza (CMS, CMSR, and CMR) were grouped in the same clade (hierarchical clustering for samples) sharing this clade with rhizoplane and rhizosphere samples from Comitán (CCS and CCSR) (Fig. 3a). On the other hand, samples from the rhizosphere and rhizoplane from Todos Santos were grouped in a well-defined clade (CTS and CTSR) (Fig. 3a). And also, the root endophytic compartment samples from Comitán and Todos Santos (CCR and CTR) were grouped in another clade (Fig. 3a). These last clustering results followed the same trend applying principal components (PCA) and principal coordinates (PCoA) analyses (Fig. 4a, b).

The root endophytic compartment samples, rhizosphere samples, and rhizoplane samples from $S$. lycopersicum crop fields were assessed through beta-diversity analysis to determine differences between samples. All samples from $S$. lycopersicum crop fields were analyzed by a hierarchical clustering analysis based on a BrayCurtis distance estimation matrix. This analysis showed that microbial community structure for rhizosphere samples and root endophytic compartment samples were similar, in spite of the different crop field location they came from, except in the case of root endophytic compartment sample from La Matanza (TMR), which was grouped with the rhizoplane samples (TPS, TLS, TMS) (Fig. 2b). Similar results were obtained after an unsupervised hierarchical biclustering analysis of the most abundant OTUs (> 100 reads/sample) (Fig. 3b). When PCA and PCoA analyses were applied, we were able to determine, as well as hierarchical clustering based on Bray-Curtis distance estimation matrix and unsupervised hierarchical biclustering analyses, that the microbial community structures were independent of the tomato crop field location (Fig. 4c, d). Besides, we carried out a permutational analysis of variance (PERMANOVA) to determine whether the type of sample (rhizoplane, rhizosphere, and root endophytic compartment) and crop a)

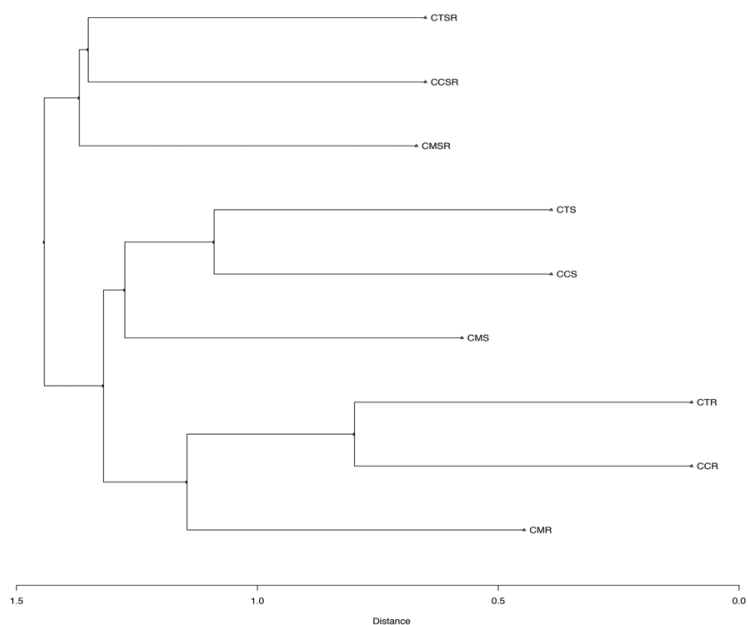

b)

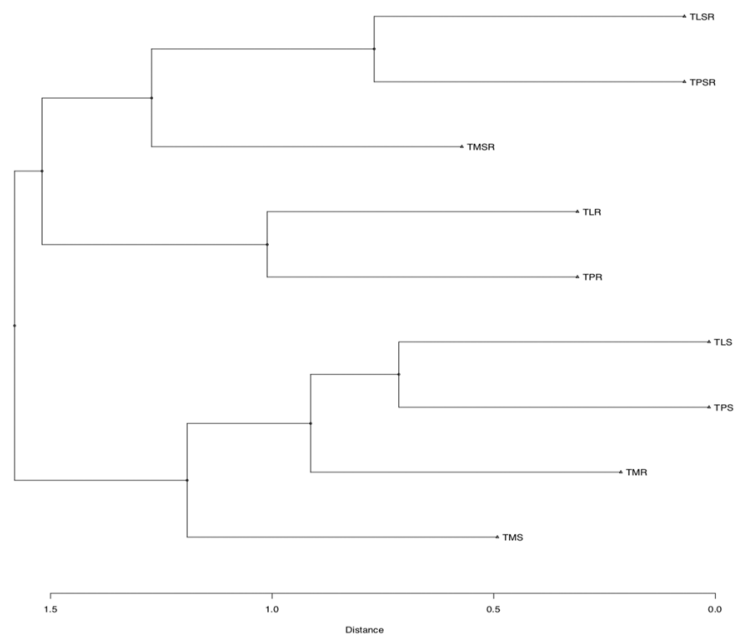

Fig. 2 Analysis of the Bray-Curtis beta-diversity index in the rhizosphere, rhizoplane, and root endophytic compartment of a C. annuum and $\mathbf{b}$ S. Iycopersicum 
a)

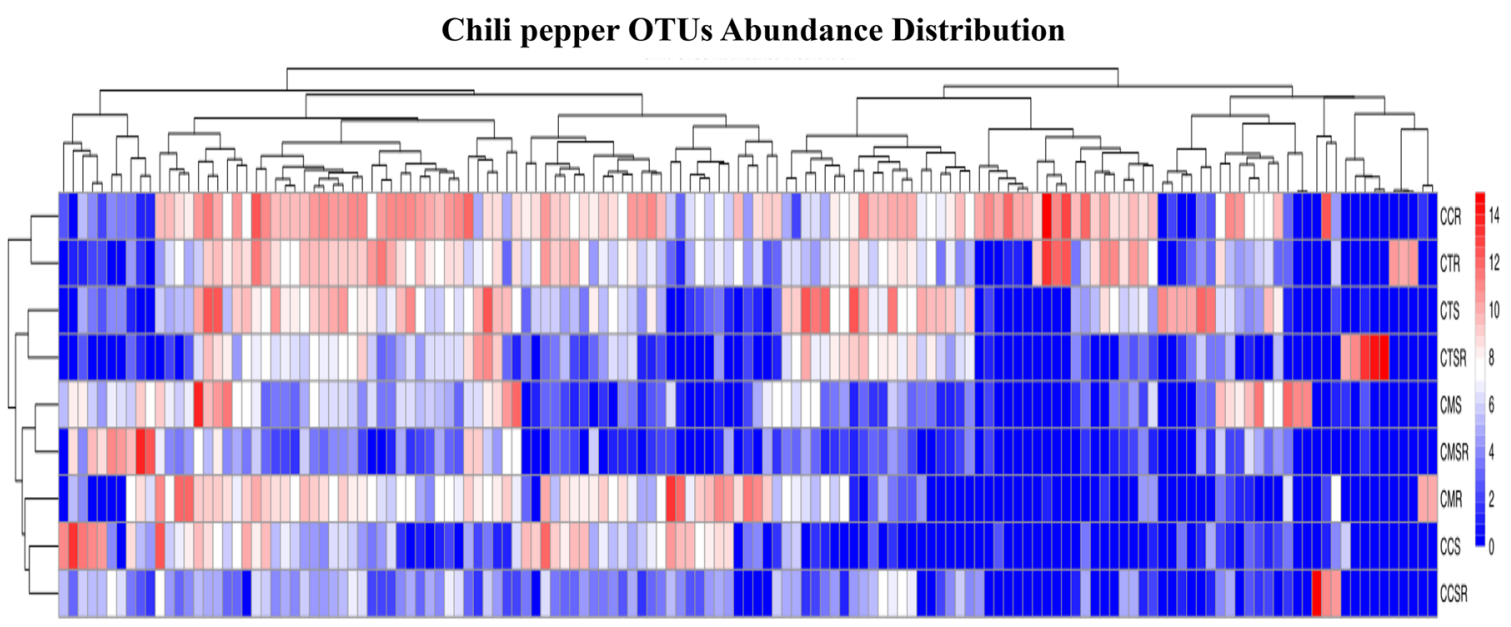

b)

Tomato OTUs Abundance Distribution

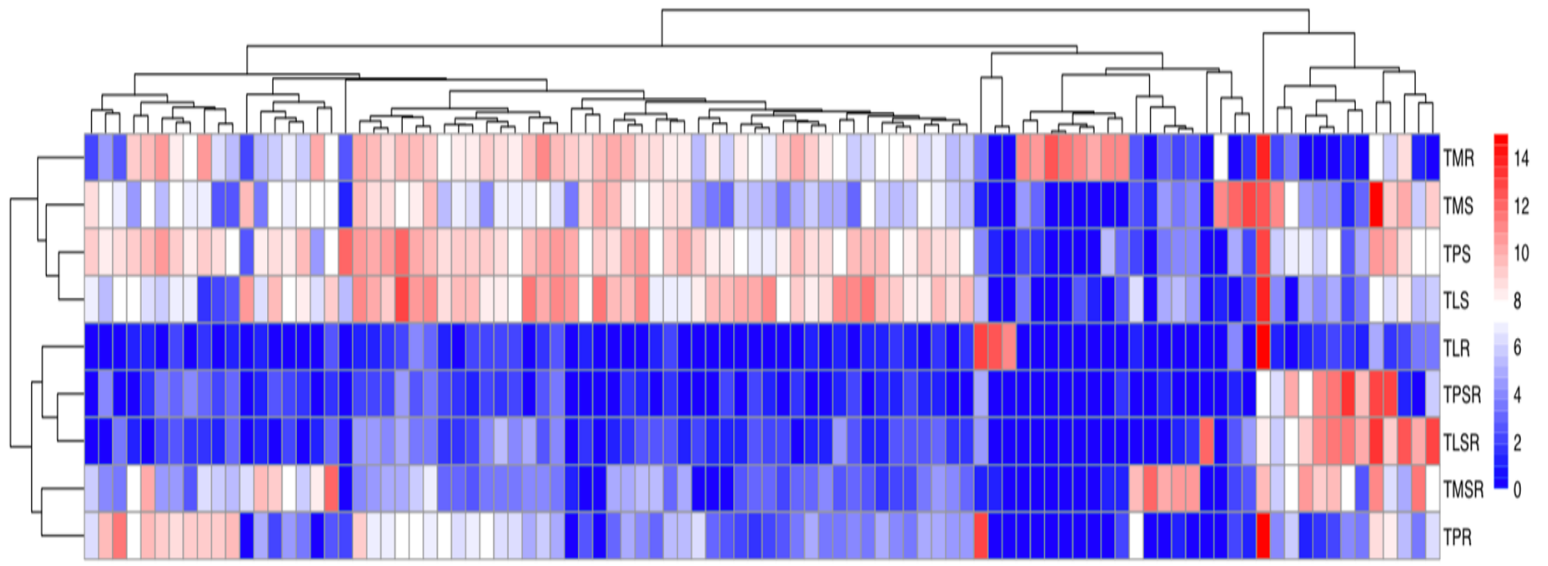

Fig. 3 Distribution analysis of the abundance of the OTUs found in a C. annuum and b S. lycopersicum

plant species affected the bacterial community structures. PERMANOVA confirmed that the type of sample was the main factor (crop plant species was not statistically significant) that influenced bacterial community structures $\left(R^{2}=0.18245, P=0.003\right)$.

\section{Composition of microbial communities associated with $C$.}

\section{annuum and S. lycopersicum}

The microbial communities associated with $C$. annuum and $S$. lycopersicum in the rhizosphere, rhizoplane, and root endophytic compartment were analyzed. The chili pepper microbial community structures for the rhizoplane from the three crop field locations (CTS, CCS, and CMS) were mainly composed of the bacterial phyla of Proteobacteria (up to 64.45\%), Firmicutes (up to 15.55\%), and Actinobacteria (up to 10.87\%) (Fig. 5a). The most abundant classes were Gammaproteobacteria (up to 29.14\%), Betaproteobacteria (up to 20.50\%), Alphaproteobacteria (up to 12.46\%), and Bacilli (up to 12.75\%)
(Fig. 5b). The most abundant families were Pseudomonadaceae (up to 12.63\%), Enterobacteriaceae (up to $11.70 \%$ ), Burkholderiaceae (up to 11.35\%), and Bacillaceae (up to 3.85\%) (Fig. 5c), and from the Pseudomonadaceae family, the genus Pseudomonas (up to 10.34\%) was the most abundant (Fig. S2b). The rhizosphere microbial community structures were mainly composed of the bacterial phyla of Firmicutes (up to 77.79\%), Cyanobacteria (up to 61.22\%), and Proteobacteria (up to 22.65\%) (CCSR, CMSR, and CTSR) (Fig. 5a). The most abundant classes were Bacilli (up to 26.39\%), Clostridia (up to $22.66 \%$ ), Alphaproteobacteria (up to $5.26 \%$ ), Betaproteobacteria (up to 8.14\%), and Gammaproteobacteria (up to 8.61\%) (Fig. 5b). The main families were Aerococcaceae (up to 25.74\%), Clostridiaceae (up to 21.29\%), Enterobacteriaceae (up to 5.45\%), Chromobacteriaceae (up to 4.57\%), and Rhizobiaceae (up to 1.45\%) (Fig. 5c). The root endophytic compartment microbial community structures were mainly composed of the bacterial phyla 


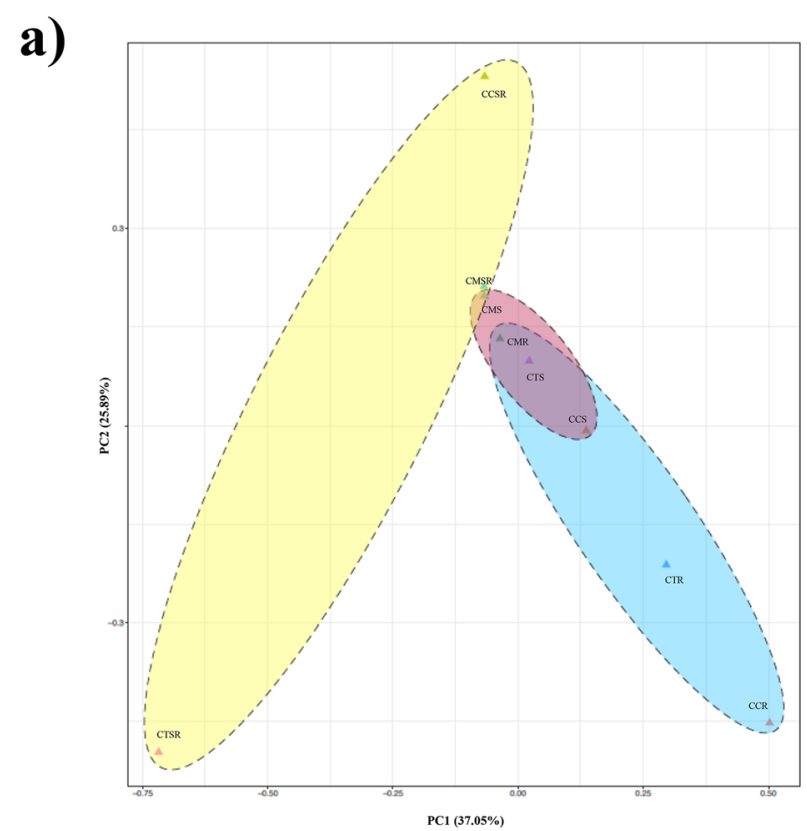

\section{c)}

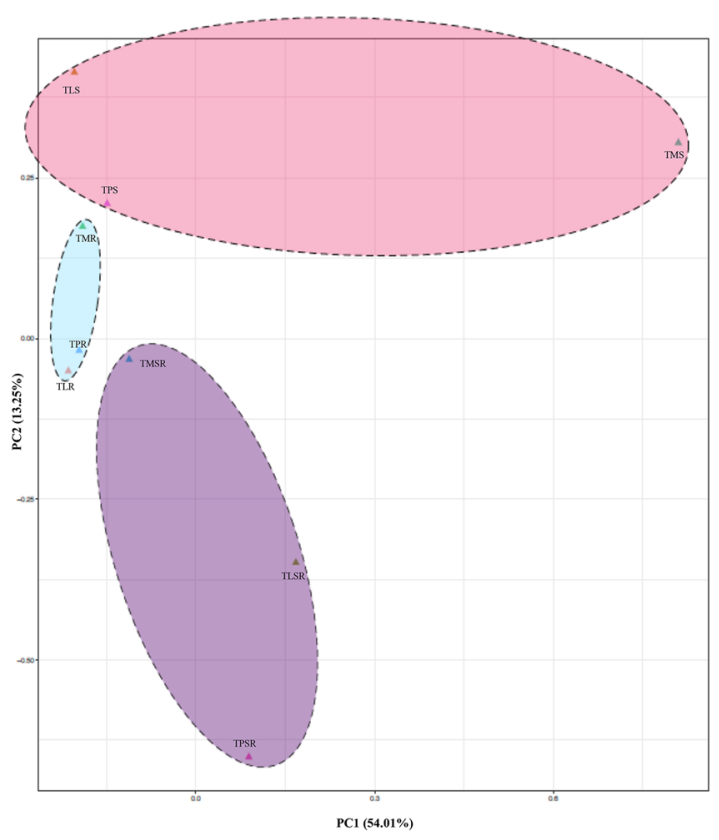

b)

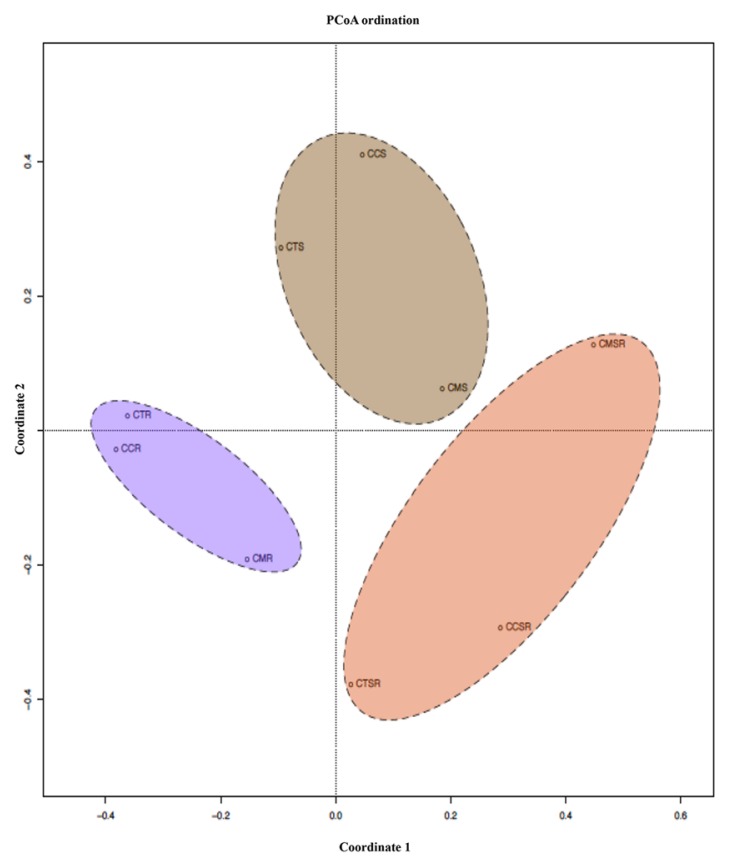

d)

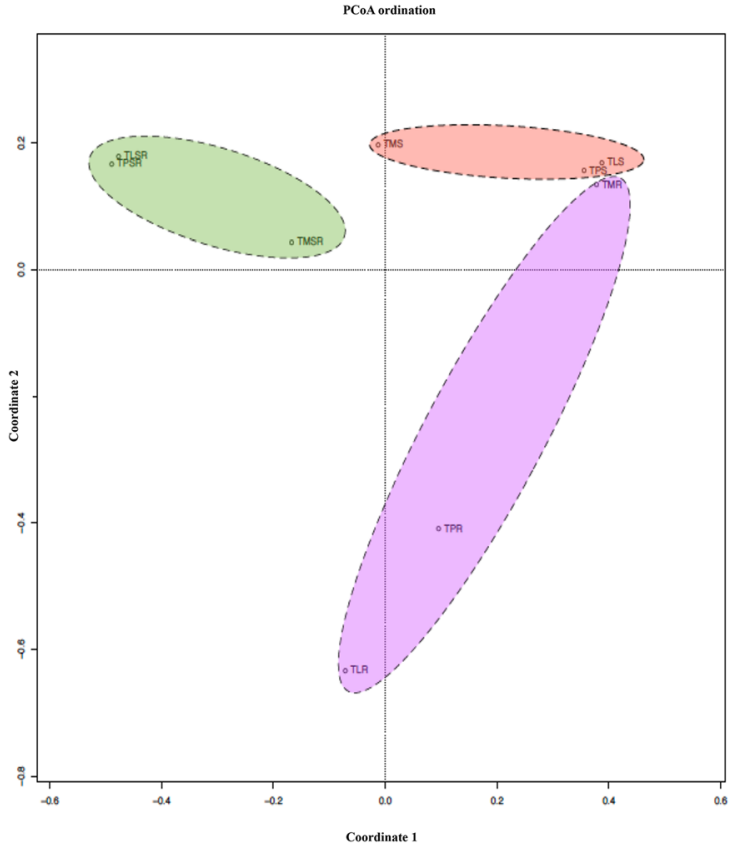

Fig. 4 Principal component (PCA) and principal coordinate (PCOA) analyses of the UniFrac distances of bacterial communities in the different samples of C. annuum, and S. Iycopersicum. a PCA and $\mathbf{b}$ PCoA of the bacterial communities from C. annuum. $\mathbf{c}$ PCA and $\mathbf{d}$ PCoA of the bacterial communities from S. lycopersicum

Proteobacteria (up to $45.23 \%$ ), Firmicutes (up to $17.85 \%$ ), Bacteroidetes (up to 12.46\%), Actinobacteria (up to 9.82\%), and Acidobacteria (up to 4.06\%) (CTR, CCR, and CMR) (Fig. 5a). The most abundant classes were Alphaproteobacteria (up to 23.06\%), Betaproteobacteria (up to $12.15 \%$ ), Clostridia (up to $12.47 \%$ ), Gammaproteobacteria (up to $8.33 \%$ ), Bacilli (up to 4.30\%), and Chitinophagia (up to 5.42\%) (Fig. 5b). The main families were Thermoanaerobacteraceae (up to $11.65 \%$ ), Chitinophagaceae (up to $5.42 \%$ ), and Rhizobiaceae (up to $4.29 \%$ ) (Fig. 5c).

The microbial communities associated with S. lycopersicum in the rhizosphere, rhizoplane, and root endophytic compartment were analyzed. The microbial community structures from rhizosphere were dominated almost exclusively by Proteobacteria (up to 99.33\%) and 


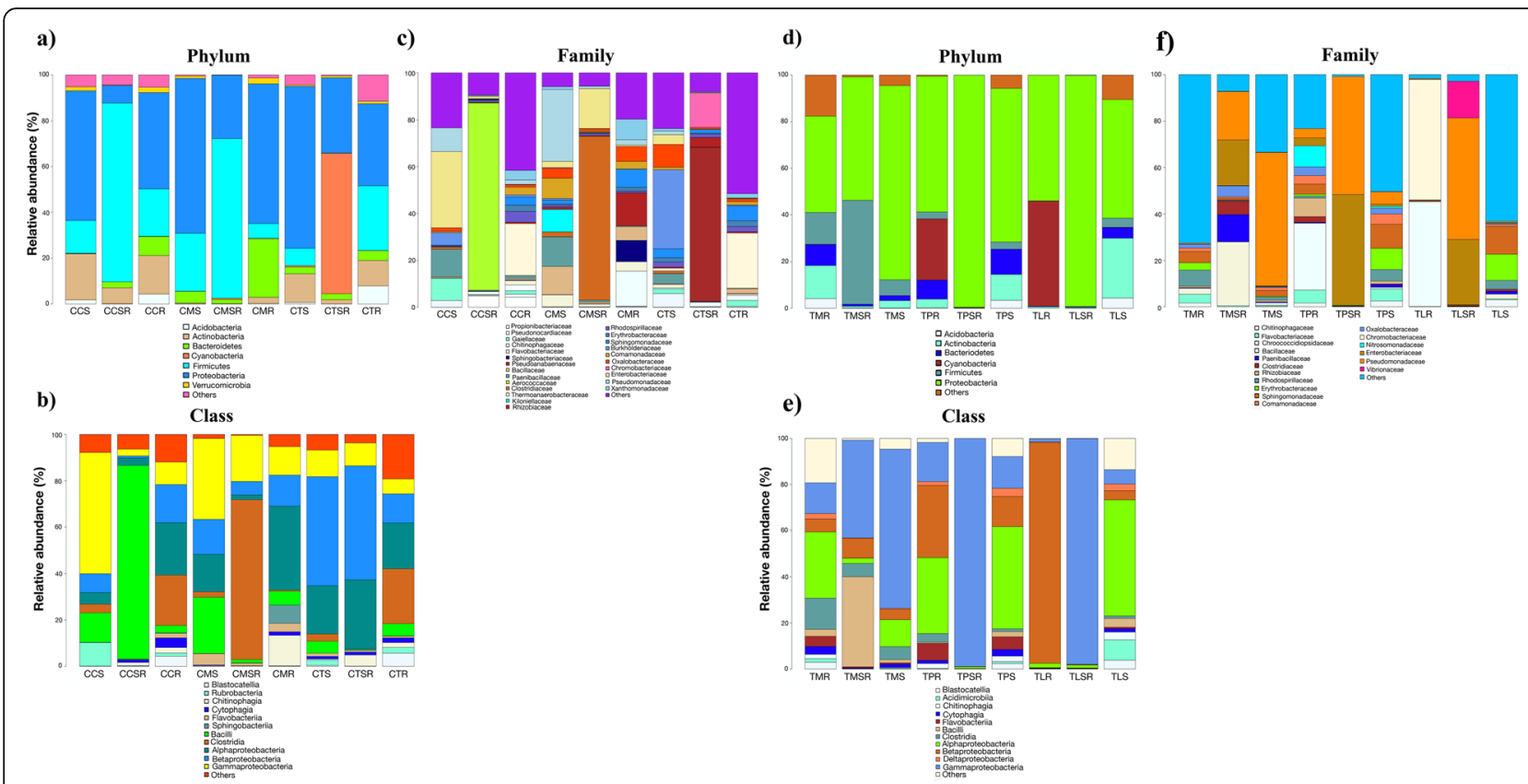

Fig. 5 Profile of bacterial communities from C. annuum and S. lycopersicum. Dominant phylum, classes, and families in C. annuum (a-c) and S. lycopersicum $(\mathbf{d}-\mathbf{f})$.

Firmicutes (up to $44.40 \%$ ) (Fig. 5d); two classes, from these phyla, were the main components of the rhizosphere: Gammaproteobacteria (up to 79.42\%) and Bacilli (up to 12.91\%) (Fig. 5e). Moreover, the most abundant families were Pseudomonadaceae (up to $38.81 \%$ ), Enterobacteriaceae (up to $30.37 \%$ ), and Bacillaceae (up to 8.84\%) (Fig. 5f), and the most represented genera were Pseudomonas and Bacillus (TPSR, TLSR, and TMSR). The rhizoplane microbial community structures were mainly composed of Proteobacteria (up to 66.11\%), Actinobacteria (up to $13.17 \%$ ), Bacteroidetes (up to 5.74\%), and Firmicutes (up to 4.57\%) (Fig. 5d). The main classes and families that we found were those belonging to the Proteobacteria phylum: Alphaproteobacteria (up to 29.76\%), Gammaproteobacteria (up to 27.69\%), and Betaproteobacteria (up to 6.25\%) (Fig. 5e), and Pseudomonadaceae (up to 19.33\%), Sphingomonadaceae (up to 7.24\%), and Erythrobacteraceae (up to 5.92\%), respectively (Fig. 5f), and the most abundant genus was Pseudomonas (TLS, TPS, and TMS). The microbial community structures from root endophytic compartment samples were dominated by Proteobacteria (up to 56.07\%), Firmicutes (up to $13.51 \%$ ), Actinobacteria (up to $6.15 \%$ ), and Bacteroidetes (up to 5.70\%) (Fig. 5d). The most abundant classes were Betaproteobacteria (up to 26.09\%), Alphaproteobacteria (up to 15.73\%), and Clostridia (up to $4.50 \%$ ) (Fig. 5e). And the most abundant families we found were Chrooccidiopsidaceae (up to 23.72\%), Chromobacteriaceae (up to 17.25\%), Rhodospirillaceae (up to $2.38 \%$ ), and Sphingomonadaceae (up to 2.82\%) (Fig. 5f).

\section{Comparison of bacterial communities associated with the} Solanaceae family

To determine which OTUs were shared and exclusive for rhizoplane, rhizosphere, and root endophytic compartment between the two plant Solanaceae species ( $C$. annuum and $S$. lycopersicum), we performed several comparative analyses. We proceeded to carry out the 2628 OTU comparison between plant Solanaceae species, for which 573 OTUs were exclusive in S. lycopersicum, 521 OTUs were exclusive for C. annuum, and 1534 OTUs were shared for both species (Fig. 6a). Next, the comparison was carried out from 2032 OTUs found between the rhizoplane of $C$. annuum and the rhizoplane of S. lycopersicum, for which 1032 OTUs were found exclusively in S. lycopersicum rhizoplane, 204 OTUs were exclusive for $C$. annuum rhizoplane, and 796 OTUs were shared for the rhizoplane of both plant Solanaceae species (Fig. 6b). The comparison between the 1243 OTUs found for C. annuum rhizosphere and S. lycopersicum rhizosphere was performed, for which 457 OTUs were exclusively in C. annuum rhizosphere, 292 OTUs were found exclusively in S. lycopersicum rhizosphere, and 494 OTUs were shared for the rhizosphere of both plant Solanaceae species (Fig. 6c). Finally, the comparison between the 1978 OTUs found for C. annuum root endophytic compartment and $S$. lycopersicum root endophytic compartment was performed, for which we found that 705 OTUs were exclusive for $C$. annuum root endophytic compartment, 294. OTUs were exclusive for S. lycopersicum root endophytic compartment, and 979 


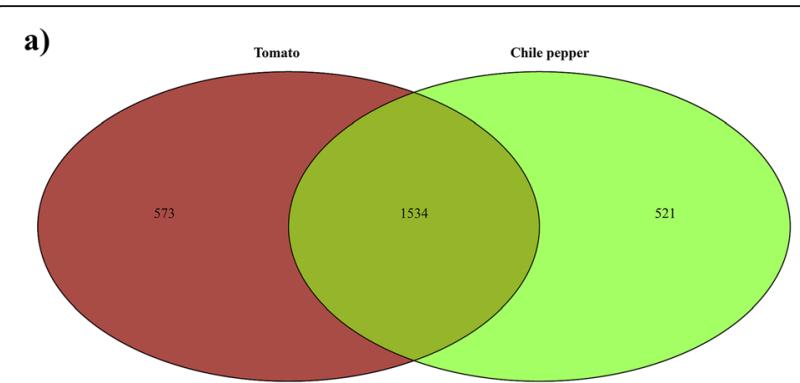

b)

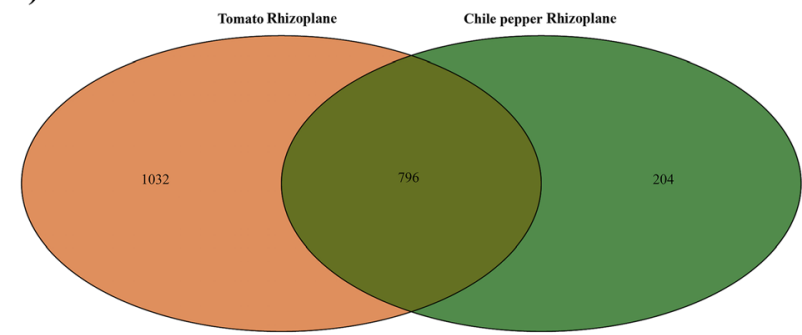

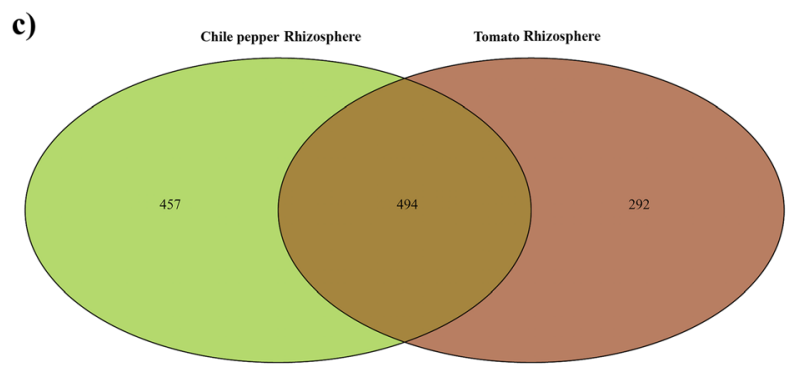

d)

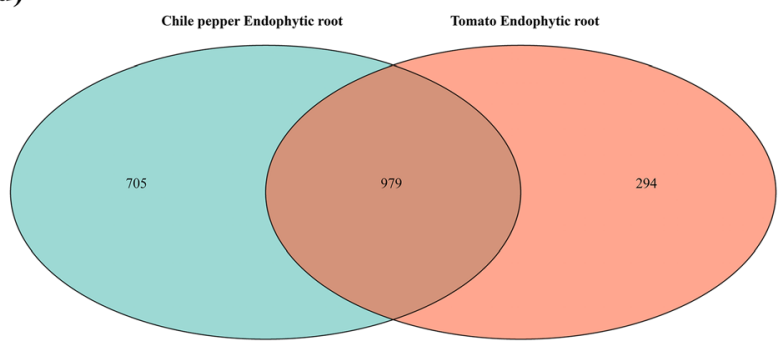

Fig. 6 Analysis of the Bray-Curtis beta-diversity index in the Solanaceae family in a both species, $\mathbf{b}$ rhizoplane, $\mathbf{c}$ rhizosphere, and $\mathbf{d}$ endophytic root samples

OTUs were shared for the root endophytic compartment of both plant Solanaceae species (Fig. 6d).

Furthermore, when we analyzed the core microbiome (OTUs present in the range from 50 to $75 \%$ of the samples) for both plant Solanaceae species, we found that 462 OTUs were present in at least $50 \%$ of the samples, while 199 were present in at least $75 \%$ of the samples. Finally, 28 OTUs were present in $100 \%$ of the samples. It is worth noting that the main bacterial class was the Gammaproteobacteria, which comprises the Pseudomonadaceae family, and the genus Pseudomonas, followed by Rhizobiales, Rhodospirillales, and Sphingomonadales (Table S3).

\section{Differentially abundant OTU analysis in the Solanaceae family}

To identify the OTUs that were depleted or enriched in the samples of rhizoplane, rhizosphere, and root endophytic compartment in C. annuum and S. lycopersicum, the total number of differentially abundant OTU (DAOTU) analysis was made together as Solanaceae family. This test permits to determine the changes that occurred in the bacterial community composition for the Solanaceae family, between the different types of samples analyzed. DAOTU analysis was made comparing OTU proportion in the rhizoplane as a control against root endophytic compartment or rhizosphere samples. The total number of DAOTUs (enriched or depleted) was greater in the root endophytic compartment (33 OTUs) than in the rhizosphere samples (20 OTUs) (Fig. 7). In the rhizosphere, the enriched OTUs included taxa from Proteobacteria (16 OTUs) and Bacteroidetes (1 OTU) (Fig. 7a). Enriched Proteobacteria and
Bacteroidetes OTUs were belonging to Chitinophagaceae (1 OTUs), Pseudomonadaceae (3 OTUs), Erwiniaceae (1 OTUs), Enterobacteriaceae (5 OTUs), Burkholderiales (2 OTUs), Rhizobiaceae (2 OTUs), Hyphomicrobiaceae (2 OTUs), and Caulobacteraceae (1 OTUs). In contrast, 3 OTUs were depleted in the rhizosphere. The depleted OTUs included taxa from Proteobacteria (Oxalobacteraceae, 1 OTU), Firmicutes (Thermoanaerobacteraceae, 1 OTU), and Tenericutes (Mycoplasmataceae, 1 OTU) (Fig. 7a). Otherwise, in the case of root endophytic compartment, 18 OTUs were significantly enriched (Fig. 7b). OTUs included taxa from Proteobacteria (6 OTUs), Actinobacteria (4 OTUs), Firmicutes (3 OTUs), Bacteroidetes (2 OTUs), Caldiserica (2 OTUs), and Planctomycetes (1 OTU). The enriched OTUs were belonging to families Streptomycetaceae (1 OTU), Pseudonocardiaceae (1 OTU), Microbacteriaceae (2 OTUs), Cytophagaceae (1 OTU), Chitinophagaceae (1 OTUs), Caldisericaceae (2 OTUs), Thermoanaerobacteraceae (2 OTUs), Gracilibacteraceae (1 OTU), Gemmataceae (1 OTU), Enterobacteriaceae (2 OTUs), Burkholderiales (2 OTUs), and Comamonadaceae (2 OTUs). Finally, 15 OTUs were found depleted in the root endophytic samples and included taxa from Proteobacteria (13 OTUs) and Firmicutes (2 OTUs) (Fig. 7b). Those OTUs belong to families Pseudomonadaceae (2 OTUs), Legionellaceae (1 OTU), Enterobacteriaceae (3 OTUs), Burkholderiales (6 OTUs), Phyllobacteriaceae (1 OTU), and Clostridiaceae (2 OTUs).

\section{Design in silico of biofertilizer}

The study of the core microbiome of the rhizobiome of plant species of Solanaceae family C. annuum and $S$. 


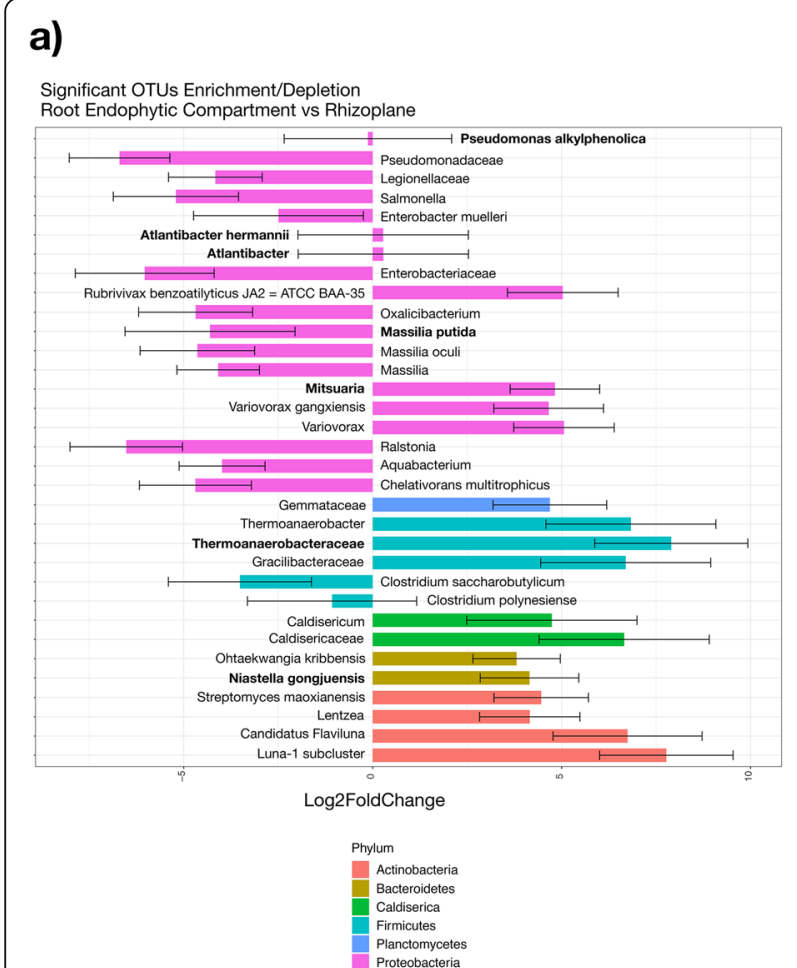

\section{b)}

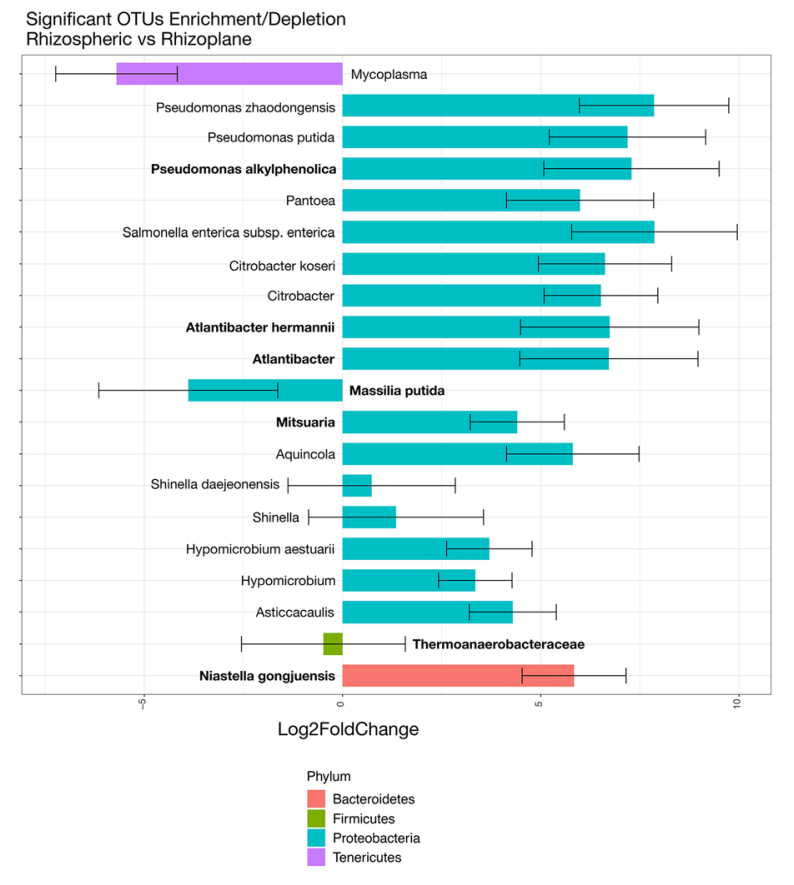

Fig. 7 Comparisons of differentially abundant OTUs. Differentially abundant OTUs (DAOTUs) were evaluated from C. annuum and S. Iycopersicum for the a root endophytic compartment and $\mathbf{b}$ rhizosphere

lycopersicum was the main objective of this work. Likewise, which bacterial group is specific for chili or tomato and which locations are specific were also objectives of this study. All of the above were for the in silico design of better biofertilizers for Solanaceae family and for species and specific crop area. At this point, with the data, we can say that a Solanaceae family-specific biofertilizer should include mainly Pseudomonas, but also Rhizobiales, Rhodospirillales, and Sphingomonadales. In the case of chili pepper, it should be composed of bacteria from families Pseudomonadaceae (in average 12.63\% OTUs), Bacillaceae (on average $2.16 \%$ OTUs), Paenibacillaceae (on average 3.08\% OTUs), or Rhodospirillaceae (in average $1.26 \%$ OTUs). For the development of biofertilizers for chili pepper specific to each area, these could be composed of bacteria from the following families: (a) Comitán: Aerococcaceae (77.23\% OTUs) and/or Thermoanaerobacteraceae (16.07\% OTUs); (b) La Matanza: Clostridiaceae (63.8\% OTUs), Rhizobiaceae (11.97\% OTUs), Chitinophagaceae (12.73\% OTUs), and/or Bacillaceae (10.83\% OTUs); and c) Todos Santos: Burkholderiaceae (28.7\% OTUs), Thermoanaerobacteraceae (18.9\% OTUs), and/or Chromobacteriaceae (13.61\% OTUs). In the case of tomato, it should be composed of bacteria from families Pseudomonadaceae (on average 19.86\% OTUs) and/or Sphingomonadaceae (on average 7.24\% OTUs). For the development of biofertilizers for tomato specific to each area, these could be composed of bacteria from the following families: (a) La Matanza: Bacillaceae (26.46\% OTUs), Rhodospirillaceae (6.23\% OTUs), and/or Sphingomonadaceae (4.17\% OTUs); (b) Pescadero: Chroococcidiopsidaceae (26.04\% OTUs); and (c) Los Planes: Chroococcidiopsidaceae (45.10\% OTUs) and/ or Chromobacteriaceae (51.76\% OTUs).

\section{Discussion}

In this study, next-generation sequencing 16S rDNA gene amplicons-based metagenomic analysis was used to characterize the core microbiome of the rhizosphere, rhizoplane, and root endophytic compartment for plant solanaceous species in arid zones, such as in the Baja California peninsula south region. This approach has the full potential to find the bacterial groups shared among the plant Solanaceae species, as well as to determine which groups are exclusive for each plant species and even to determine which bacteria might be related to plant survival and adaptation to arid environments.

By NGS sequencing, we were able to determine a greater diversity (observed OTUs and Chaol index) in root endophytic compartment samples than in rhizosphere samples for both tomato and chili pepper. These results reflected a more homogeneous composition of the bacterial communities in the rhizoplane and the rhizosphere. Bacteria with a scarce abundance in the 
rhizoplane and the rhizosphere that did not influence in the bacterial community structure probably were under the detection limit of the NGS technique, while in the root, this limitation was surpassed by abundance of nutrients that favored the enrichment (proliferation) of those bacteria in this organ. Interestingly rhizoplane samples from tomato and chili pepper crop fields showed a contrasting and specific behavior for each crop. It is worth noting that a limited (or a few) number of studies have been addressed to explore bacterial communities associated with these two plants, despite their agricultural and economic importance. Tian et al. (2015) determined a greater bacterial diversity and richness (observed OTUs and Chaol, and Shannon index) in rhizoplane microbial communities than those for root endophytic compartments. Endophytic bacteria have been considered a subset of rhizospheric bacteria, with the advantage to be in close contact with the plant host for a constant supply of nutrients (Afzal et al. 2019). The results between both studies in tomato-related microbial communities are quite remarkable, since both studies were conducted independently and in the most contrasting conditions (greenhouse conditions for Tian et al. (2015) and crop fields in arid zones for our study). Those results suggest a tightly and broad regulation of the surrounding microbiota exerted by tomato plants, in spite of the environmental conditions, which enhance the plant survival.

When we analyzed the differences/similitudes between the rhizosphere, rhizoplane, and root endophytic compartment samples for both plant Solanaceae species through beta-diversity indices (Bray-Curtis distance matrix, hierarchical clustering analysis, PCoA, and PCA analysis), the grouping of the samples followed a trend. That can be clustered by the type of sample (rhizosphere, rhizoplane, and root endophytic compartment). Besides, the alpha indices showed the influence of the location on the diversity (observed OTUs and Chao1 index), since the samples that were collected from the crop fields nearer to the shores showed the greatest diversity indices. These results were quite similar for previous reports that the crop field location exerted a significant effect on crop-associated bacterial assemblages (Allard et al. 2016; Rashid et al. 2012).

The bacterial community structures for all types of samples analyzed and for chili pepper were dominated by the Proteobacteria and Firmicutes phyla, and the main classes were Alphaproteobacteria, Betaproteobacteria, Gammaproteobacteria, and Bacilli. Interestingly, the family relative abundances showed specific differences for each type of sample: Xanthomonadaceae, Bacillaceae, and Burkholderiaceae were among the most abundant families for rhizoplane samples; Aerococcaceae, Clostridiaceae, and Chromobacteriaceae for rhizosphere samples; and Thermoanaerobacteraceae for root endophytic compartment samples. However, several abundant families were shared between the type of samples, such as Enterobacteriaceae, Pseudomonadaceae, and Rhizobiaceae. Other previous reports have found that the most abundant bacterial phyla for rhizoplane, rhizosphere, and root endophytic compartment samples were Proteobacteria, Bacteroidetes, Actinobacteria, and Firmicutes (Rasche et al. 2006; Kolton et al. 2011; Marasco et al. 2012; Asaff-Torres et al. 2017; Ros et al. 2017). Finally, in the case of root endophytic compartment samples, this is the first report for chili pepper describing the microbial community structures applying nextgeneration sequencing techniques.

The tomato samples for rhizoplane, rhizosphere, and root endophytic compartment were composed mainly by the Proteobacteria phylum and, to a lesser extent, by Firmicutes, Actinobacteria, and Bacteroidetes. The dominant classes for all samples were Alphaproteobacteria, Betaproteobacteria, and Gammaproteobacteria, and the most abundant families were Pseudomonadaceae and Sphingomonadaceae, which also belong to the Proteobacteria phylum. Other previous reports have been shown that the Proteobacteria phylum and the Pseudomonadaceae family were the most representative OTUs for all tomato-related samples (Ottese et al. 2013; Ottese et al. 2015; Tian et al. 2015; Kalam et al. 2016; Pii et al. 2016; Larousse et al. 2017; Qiao et al. 2017).

The comparative analysis of the bacterial communities related to both plant Solanaceae species unveiled the remarkable feature of the high proportion of shared microbiome among these plants. Interestingly, the DAOTU analysis showed a contrasting behavior among rhizosphere and root endophytic compartment samples for the enrichment and depletion of OTUs belonging to Pseudomonadaceae, Enterobacteriaceae, and Burkholderiales, respectively. This reflects the selectivity exerted by the plant Solanaceae species and translated into a high proportion of the shared microbiome. This feature reflects that both plant Solanaceae species have similar ecological, and even physiological, requirements leading to select specific bacteria, or Solanaceae-related bacteria, to address their needs. Furthermore, from the core microbiome analysis for both plant Solanaceae species and related samples (rhizoplane and rhizosphere) taking the OTU presence in at least $50 \%, 75 \%$, and $100 \%$ (absolute) of the samples, we found that the Pseudomonadaceae family and the genus Pseudomonas were present at $100 \%$ of the samples, and this genus was also the most abundant in the core microbiome at $100 \%$ criteria. Previous reports that analyzed the microbiome associated to plant Solanaceae also found that the genus Pseudomonas was among the most abundant genera, and the presence of this genus has been related directly with an incidence 
reduction of certain diseases caused by phytopathogenic fungi (Ottese et al. 2013; Hu et al. 2016).

Finally, improved formulations of the biofertilizers are necessary for creating and commercializing new biofertilizers that are more effective, stable, and of better quality (Bashan et al. 2014; Mahanty et al. 2017). Initially, studies to characterize plant-associated communities relied on cultivation-based methods. Massive parallel sequencing has dramatically improved our ability to identify and quantify community members (Lebeis 2014). At this point, using the next-generation sequencing technologies for the in silico design of biofertilizers that can be developed from the bacterial communities of each species of crop and area to be cultivated, such as those proposed in this work, could accelerate the obtaining of better ecofriendly biofertilizers that help to increase agricultural production without impacting the environment.

\section{Conclusion}

In our study, we were able to define by NGS coupled with metagenomic analysis of the core microbiome in the rhizobiome (rhizosphere, rhizoplane, and root endophytic compartment) of plant Solanaceae species in several crop fields from arid zones. We found that the Proteobacteria was the main phylum in the rhizobiome for both plant Solanaceae species. The crop field location (near the shores) had a direct and positive impact on bacterial diversity. Finally, the Proteobacteria phylum was represented mainly by the Pseudomonadaceae family and the Pseudomonas genus, which also were present for all the samples, and in turn, these integrate the core microbiome in the rhizobiome of these two plants belonging to the Solanaceae family.

\section{Supplementary information}

Supplementary information accompanies this paper at https://doi.org/10. 1186/s13213-020-01572-x.

Additional file 1: Figure S1. Alpha-diversity indices of bacterial communities across the different localities of a) C. annuum, and b) $S$. lycopersicum.

Additional file 2: Figure S2. Profile of bacterial communities from C. annuum and S. lycopersicum. Dominant order and genus in C. annuum (a and b), and S. lycopersicum (c and d).

Additional file 3: Figure S3. Alfa index between C. annuum, and S. lycopersicum.

Additional file 4: Figure S4. Alfa index between C. annuum, and S. lycopersicum by type of sample.

Additional file 5: Figure S5. Alfa index in the Solanaceae family by type of sample.

Additional file 6: Figure S6. Alfa index in the Solanaceae family by sampling site.

Additional file 7: Table S1. Alpha-diversity indices of bacterial communities across the different crop field locations. Table S2. Alpha-diversity indices of bacterial communities across the different localities by type of sample. Table S3. OTUs present in the $100 \%$ of the samples.

\section{Acknowledgements}

Current investigations from the group are supported by CONACYT/México. We thank the technical assistance to Ángel Edgardo Carrillo-García, Saúl Edel Briseño Ruiz, Ariel Arturo Cruz Villlacorta, and Patricia Hinojosa Baltazar.

\section{Authors' contributions}

Aarón Barraza: Investigation, Methodology, Formal analysis, Writing -original draft. Goretty Caamal-Chan: Investigation, Writing - review \& editing. Thelma

Castellanos: Funding acquisition, Writing - review \& editing. Abraham Loera-Muro Conceptualization, Formal analysis, Writing - original draft, review \& editing, Project administration. The author(s) read and approved the final manuscript.

\section{Funding}

The current investigation was supported by CONACYT/Mexico through the funds provided to CIBNOR.

\section{Ethics approval and consent to participate}

N/A

\section{Consent for publication}

$\mathrm{N} / \mathrm{A}$

Competing interests

The authors declare that there are no competing interest.

\section{Author details}

${ }^{1}$ CONACYT-Centro de Investigaciones Biológicas del Noroeste, SC, Instituto Politécnico Nacional 195, Playa Palo de Santa Rita Sur, C.P. 23096, La Paz, B.C.S., Mexico. ${ }^{2}$ Centro de Investigaciones Biológicas del Noroeste, SC, Instituto Politécnico Nacional 195, Playa Palo de Santa Rita Sur, La Paz, B.C.S. C.P. 23096 " Mexico.

Received: 11 September 2019 Accepted: 23 April 2020

Published online: 29 May 2020

\section{References}

Afzal I, Shinwari ZK, Sikandar S, Shahzad S (2019) Plant beneficial endophytic bacteria: mechanisms, diversity, host range and genetic determinants. Microbiol Res 221:36-49. https://doi.org/10.1016/j.micres.2019.02.001

Allard SM, Walsh CS, Wallis AE, Ottesen AR, Brown EW, Micallef SA (2016) Solanum lycopersicum (tomato) hosts robust phyllosphere and rhizosphere bacterial communities when grown in soil amended with various organic and synthetic fertilizers. Sci Total Environ 573:555-563. https://doi.org/10. 1016/j.scitotenv.2016.08.157

Asaff-Torres A, Armendáriz-Ruiz M, Kirchmayr M, Rodríguez-Heredia R, Orozco M, Mateos-Díaz JC, Figueroa-Yáñez L, Baqueiro-Peña I, Verdín J (2017) Rhizospheric microbiome profiling of Capsicum annuum L. cultivated in amended soils by 165 and internal transcribed spacer 2 rRNA amplicon metagenome sequencing. Genome Announc 5:e00626-e00617. https://doi. org/10.1128/genomeA.00626-17

Ávila MP, Oliveira-Junior ES, Reis MP, Hester ER, Diamantino C, Veraart AJ, Lamers LPM, Kosten S, Nascimento AMA (2019) The water hyacinth microbiome: link between carbon turnover and nutrient cycling. Microb Ecol. https://doi.org/ 10.1007/s00248-019-01331-9

Barchenger DW, Lamour KH, Bosland PW (2018) Challenges and strategies for breeding resistance in Capsicum annuum to the multifarious pathogen, Phytophthora capsici. Front Plant Sci 9:628. https://doi.org/10.3389/fpls.2018. 00628

Bashan Y, de Bashan LE, Prabhu SR, Hernandez JP (2014) Advances in plant growth-promoting bacterial inoculant technology: formulations and practical perspectives (1998-2013). Plant Soil 378:1-33

Camilios-Neto D, Bonato P, Wassem R, Tadra-Sfeir MZ, Brusamarello-Santos LC, Valdameri G, Donatti L, Faoro H, Weiss VA, Chubatsu LS, Pedrosa FO, Souza EM (2014) Dual RNA-seq transcriptional analysis of wheat roots colonized by Azospirillum brasilense reveals up-regulation of nutrient acquisition and cell cycle genes. BMC Genomics 15:378. https://doi.org/10.1186/1471-2164-15-378

Castañeda LE, Barbosa O (2017) Metagenomic analysis exploring taxonomic and functional diversity of soil microbial communities in Chilean vineyards and surrounding native forests. PeerJ 5:e3098. https://doi.org/10.7717/peerj.3098

Coleman-Derr D, Desgarennes D, Fonseca-Garcia C, Gross S, Clingenpeel S, Woyke T, North G, Visel A, Partida-Martinez LP, Tringe SG (2016) Plant 
compartment and biogeography affect microbiome composition in cultivated and native agave species. New Phytol 209:798-811. https://doi. org/10.1111/nph.13697 Epub 2015 Oct 15

Desgarennes D, Garrido E, Torres-Gomez MJ, Peña-Cabriales JJ, Partida-Martinez LP (2014) Diazotrophic potential among bacterial communities associated with wild and cultivated agave species. FEMS Microbiol Ecol 90:844-857. https://doi.org/10.1111/1574-6941.12438

Fonseca-García C, Coleman-Derr D, Garrido E, Visel A, Tringe SG, Partida-Martínez LP (2016) The cacti microbiome: interplay between habitat-filtering and hostspecificity. Front Microbiol 7:150. https://doi.org/10.3389/fmicb.2016.00150

Haak DC, Kostyun JL, Moyle LC (2014) Merging ecology and genomics to dissect diversity in wild tomatoes and their relatives. Adv Exp Med Biol 781:273-298. https://doi.org/10.1007/978-94-007-7347-9_14

Hu J, Wei Z, Friman VP, Gu SH, Wang XF, Eisenhauer N, Yang TJ, Ma J, Shen QR, Xu YC, Jousset A (2016) Probiotic diversity enhances rhizosphere microbiome function and plant disease suppression. MBio. 7:e01790-e01716. https://doi. org/10.1128/mBio.01790-16

Kalam S, Das SN, Basu A, Podile AR (2017) Population densities of indigenous Acidobacteria change in the presence of plant growth promoting rhizobacteria (PGPR) in rhizosphere. J Basic Microbiol 57:376-385. https://doi. org/10.1002/jobm.201600588

Kolton M, Meller Harel Y, Pasternak Z, Graber ER, Elad Y, Cytryn E (2011) Impact of biochar application to soil on the root-associated bacterial community structure of fully developed greenhouse pepper plants. Appl Environ Microbiol 77:4924-4930. https://doi.org/10.1128/AEM.00148-11

Larousse M, Rancurel C, Syska C, Palero F, Etienne C, Industri B, Nesme X, Bardin M, Galiana E (2017) Tomato root microbiota and Phytophthora parasitica-associated disease. Microbiome 5:56. https://doi.org/10.1186/ s40168-017-0273-7

Lebeis SL (2014) The potential for give and take in plant-microbiome relationships. Front Plant Sci 5:287. https://doi.org/10.3389/fpls.2014.00287

Lopez BR, Bashan Y, Bacilio M (2011) Endophytic bacteria of Mammillaria fraileana, an endemic rock-colonizing cactus of the southern Sonoran Desert. Arch Microbiol 193:527-541. https://doi.org/10.1007/s00203-011-0695-8

Mahanty T, Bhattacharjee S, Goswami M, Bhattacharyya P, Das B, Ghosh A, Tribedi P (2017) Biofertilizers: a potential approach for sustainable agriculture development. Environ Sci Pollut Res Int 24:3315-3335. https://doi.org/10. 1007/s11356-016-8104-0

Manzoni S, Schimel JP, Porporato A (2012) Responses of soil microbial communities to water stress: results from a meta-analysis. Ecology 93: 930-938

Marasco R, Rolli E, Ettoumi B, Vigani G, Mapelli F, Borin S, Abou-Hadid AF, ElBehairy UA, Sorlini C, Cherif A, Zocchi G, Daffonchio D (2012) A drought resistance-promoting microbiome is selected by root system under desert farming. PLoS One 7:e48479. https://doi.org/10.1371/journal.pone.0048479

Mickan BS, Abbott LK, Solaiman ZM, Mathes F, Siddique KHM, Jenkins SN (2019) Soil disturbance and water stress interact to influence arbuscular mycorrhizal fungi, rhizosphere bacteria and potential for $\mathrm{N}$ and C cycling in an agricultural soil. Biol Fertil Soils 55:53. https://doi.org/10.1007/ s00374-018-1328-Z

Oksanen J, Blanchet F, Kindt R, Legendre P, Minchin P, et al. vegan: community ecology package. 2012.

Olanrewaju OS, Ayangbenro AS, Glick BR, Babalola OO (2018) Plant health: feedback effect of root exudates-rhizobiome interactions. Appl Microbiol Biotechnol 103:1155-1166. https://doi.org/10.1007/s00253-018-9556-6

Ottesen AR, González Peña A, White JR, Pettengill JB, Li C, Allard S, Rideout S, Allard M, Hill T, Evans P, Strain E, Musser S, Knight R, Brown E (2013) Baseline survey of the anatomical microbial ecology of an important food plant: Solanum lycopersicum (tomato). BMC Microbiol 13:114. https://doi.org/10. 1186/1471-2180-13-114

Ottesen AR, Gorham S, Pettengill JB, Rideout S, Evans P, Brown E (2015) The impact of systemic and copper pesticide applications on the phyllosphere microflora of tomatoes. J Sci Food Agric 95:1116-1125. https://doi.org/10. 1002/jsfa.7010

Passari AK, Chandra P, Zothanpuia MVK, Leo W, Gupta VK, Kumar B, Singh BP (2016) Detection of biosynthetic gene and phytohormone production by endophytic actinobacteria associated with Solanum lycopersicum and their plant-growth-promoting effect. Res Microbiol 167:692-705. https://doi.org/10. 1016/j.resmic.2016.07.001

Perveen R, Suleria HAR, Anjum FM, Butt MS, Pasha I, Ahmad S (2015) Tomato (Solanum lycopersicum) carotenoids and lycopenes chemistry; metabolism, absorption, nutrition, and allied health claims-a comprehensive review. Crit Rev Food Sci Nutr 55:919-929. https://doi.org/10.1080/10408398.2012.657809

Pii Y, Borruso L, Brusetti L, Crecchio C, Cesco S, Mimmo T (2016) The interaction between iron nutrition, plant species and soil type shapes the rhizosphere microbiome. Plant Physiol Biochem 99:39-48. https://doi.org/10.1016/j. plaphy.2015.12.002

Posada LF, Álvarez JC, Romero-Tabarez M, de Bashan L, Villegas Escobar V (2018) Enhanced molecular visualization of root colonization and growth promotion by Bacillus subtilis EA-CB0575 in different growth systems. Microbiol Res 217: 69-80. https://doi.org/10.1016/j.micres.2018.08.017

Poudel R, Jumpponen A, Kennelly MM, Rivard CL, Gomez-Montano L, Garrett KA (2019) Rootstocks shape the rhizobiome: rhizosphere and endosphere bacterial communities in the grafted tomato system. Appl Environ Microbiol 85:e01765-e01718. https://doi.org/10.1128/AEM.01765-18

Qiao J, Yu X, Liang X, Liu Y, Borriss R, Liu Y (2017) Addition of plant-growthpromoting Bacillus subtilis PTS-394 on tomato rhizosphere has no durable impact on composition of root microbiome. BMC Microbiol 17:131. Doi:1 0. 1186/s12866-017-1039-x.

Rasche F, Trondl R, Naglreiter C, Reichenauer TG, Sessitsch A (2006) Chilling and cultivar type affect the diversity of bacterial endophytes colonizing sweet pepper (Capsicum anuum L.). Can J Microbiol 52:1036-1045

Rashid S, Charles TC, Glick BR (2012) Isolation and characterization of new plant growth-promoting bacterial endophytes. Appl Soil Ecol 61:217-224

Ros M, Raut I, Santisima-Trinidad AB, Pascual JA (2017) Relationship of microbial communities and suppressiveness of Trichoderma fortified composts for pepper seedlings infected by Phytophthora nicotianae. PLoS One 12: e0174069. https://doi.org/10.1371/journal.pone.0174069

Sasse J, Martinoia E, Northen T (2018) Feed your friends: do plant exudates shape the root microbiome? Trends Plant Sci 23:25-41. https://doi.org/10.1016/j. tplants.2017.09.003

Seguí-Simarro J (2016) Androgenesis in Solanaceae. In: Methods in molecular biology, in vitro embryogenesis in higher plants. Germanà MA, Lambardi M. Springer protocols. Springer ed. New York. 209-244 pp.

SIAP, Servicio de Información Agroalimentaria y Pesquería, SAGARPA (2016) https://www.gob.mx/siap

Tian BY, Cao Y, Zhang KQ (2015) Metagenomic insights into communities, functions of endophytes, and their associates with infection by root-knot nematode, Meloidogyne incognita, in tomato roots. Sci Rep 5:17087. https:// doi.org/10.1038/srep17087

\section{Publisher's Note}

Springer Nature remains neutral with regard to jurisdictional claims in published maps and institutional affiliations.

Ready to submit your research? Choose BMC and benefit from:

- fast, convenient online submission

- thorough peer review by experienced researchers in your field

- rapid publication on acceptance

- support for research data, including large and complex data types

- gold Open Access which fosters wider collaboration and increased citations

- maximum visibility for your research: over $100 \mathrm{M}$ website views per year

At BMC, research is always in progress.

Learn more biomedcentral.com/submission 